\title{
Hereditary diffuse gastric cancer in a Japanese family with a large deletion involving $\mathrm{CDH} 1$
}

\author{
Masayoshi Yamada $\cdot$ Takeo Fukagawa $\cdot$ Takeshi Nakajima $\cdot$ Kiyoshi Asada $\cdot$ Shigeki Sekine $\cdot$ \\ Satoshi Yamashita $\cdot$ Eriko Okochi-Takada $\cdot$ Hirokazu Taniguchi $\cdot$ Ryoji Kushima $\cdot$ \\ Ichiro Oda $\cdot$ Yutaka Saito $\cdot$ Toshikazu Ushijima $\cdot$ Hitoshi Katai
}

Received: 15 May 2013/Accepted: 14 August 2013/Published online: 15 September 2013

(C) The International Gastric Cancer Association and The Japanese Gastric Cancer Association 2013

\begin{abstract}
Hereditary diffuse gastric cancer (HDGC), characterized by susceptibility to gastric signet ring cell carcinomas (SRCCs) and caused by $\mathrm{CDHI}$ germline mutations, is rare in the Japanese. We present here a Japanese family with HDGC identified by comparative genomic hybridization (CGH) analysis. A 55-year-old woman was treated with completion gastrectomy for multiple SRCCs, and pathological examination revealed approximately 200 foci of SRCC with loss of E-cadherin expression. Her 30-year-old son had surveillance endoscopy and was found to have multiple SRCCs. He underwent total gastrectomy, and 32 foci of SRCC with loss of E-cadherin expression were histologically found. Although no point
\end{abstract}

Electronic supplementary material The online version of this article (doi:10.1007/s10120-013-0298-y) contains supplementary material, which is available to authorized users.

M. Yamada · T. Nakajima $(\bowtie) \cdot$ I. Oda · Y. Saito Endoscopy Division, National Cancer Center Hospital, 5-1-1 Tsukiji, Chuo-ku, Tokyo 104-0045, Japan

e-mail: tnakajim@ncc.go.jp

M. Yamada $\cdot$ H. Taniguchi $\cdot$ R. Kushima Pathology Division, National Cancer Center Hospital, Tokyo, Japan

T. Fukagawa $\cdot$ H. Katai

Gastric Surgery Division, National Cancer Center Hospital,

Tokyo, Japan

K. Asada $\cdot$ S. Yamashita $\cdot$ E. Okochi-Takada $\cdot$ T. Ushijima Division of Epigenomics, National Cancer Center Research Institute, Tokyo, Japan

S. Sekine

Molecular Pathology Division, National Cancer Center Research Institute, Tokyo, Japan mutations were detected in $\mathrm{CDH} 1$ by sequencing, $\mathrm{CGH}$ revealed a $275-\mathrm{kb}$ deletion involving exons $7-16$ of $\mathrm{CDHI}$ in both patients. While only a few HDGCs have been reported in East Asia, patients with multiple SRCC may need to be offered appropriate genetic counseling and testing in this area.

Keywords Hereditary diffuse gastric cancer . Gastric cancer · E-cadherin · Comparative genomic hybridization

\section{Introduction}

Hereditary diffuse gastric cancer (HDGC) is an autosomal dominant disease associated with multiple signet ring cell carcinomas (SRCCs) and is caused by a germline mutation in the E-cadherin gene ( $\mathrm{CDH1})$. Guilford et al. [1] first reported that HDGC is caused by truncating mutations of $\mathrm{CDH} 1$ in New Zealand Maori families with early-onset, multiple SRCCs. In Western countries, inherited gastric cancers (GCs) are thought to account for 1-3\% of all GCs [2]. Clinically, approximately $25 \%$ of families fulfilling the criteria for the diagnosis of HDGC have inactivating $\mathrm{CDH} 1$ germline mutations [2, 3]. In contrast, in East Asian countries, including Japan, Korea, and China, with high incidences of GCs [4], HDGC has rarely been reported [5, 6]. Recently, Yamada et al. [7] reported two germline alterations in the $\mathrm{CDH} 1$ gene in two Japanese familial GCs. However, HDGC is still rarely diagnosed in East Asian countries, and it is still unknown whether HDGC is really rare or overlooked because of the high incidence of coincidental familial GC. We report here clinical characteristics and genomic analysis of a Japanese HDGC family with a $\mathrm{CDH1}$ germline mutation. 


\section{Case reports}

A 55-year-old female patient was referred to our hospital for treatment of multiple SRCCs detected by endoscopic examination during an annual health check. She had a past history of intramucosal SRCC at the age of 34 and had undergone distal gastrectomy with Billroth I reconstruction in another hospital. She had no past history of other malignancies, including lobular breast cancer. Family history of GC was noted, affecting the patient's father and paternal grandfather (Fig. 1). Esophagogastroduodenoscopy (EGD) in our hospital detected 10-12 small pale mucosal patches, mainly in the greater curvature of the remnant stomach (Fig. 2a), but no atrophic gastritis indicative of Helicobacter pylori infection. The size of each focus was endoscopically estimated as less than $10 \mathrm{~mm}$. In one of the largest lesions, narrow band imaging with magnification showed a wavy-shaped irregular microvessel pattern suggesting undifferentiated adenocarcinoma (Fig. 2b). All the biopsy specimens from six lesions demonstrated SRCC. Completion gastrectomy was performed, and the entire gastric mucosa was histologically examined.

Histopathological examination revealed approximately 200 SRCC foci, and their maximum size was $10 \mathrm{~mm}$ in diameter (Fig. 2f). None of the lesions showed submucosal invasion, and tumor cells were mostly confined to the upper mucosal layer (Fig. 2c). Periodic acid-Schiff (PAS) staining highlighted intracytoplasmic mucin in SRCC (Fig. 2d).

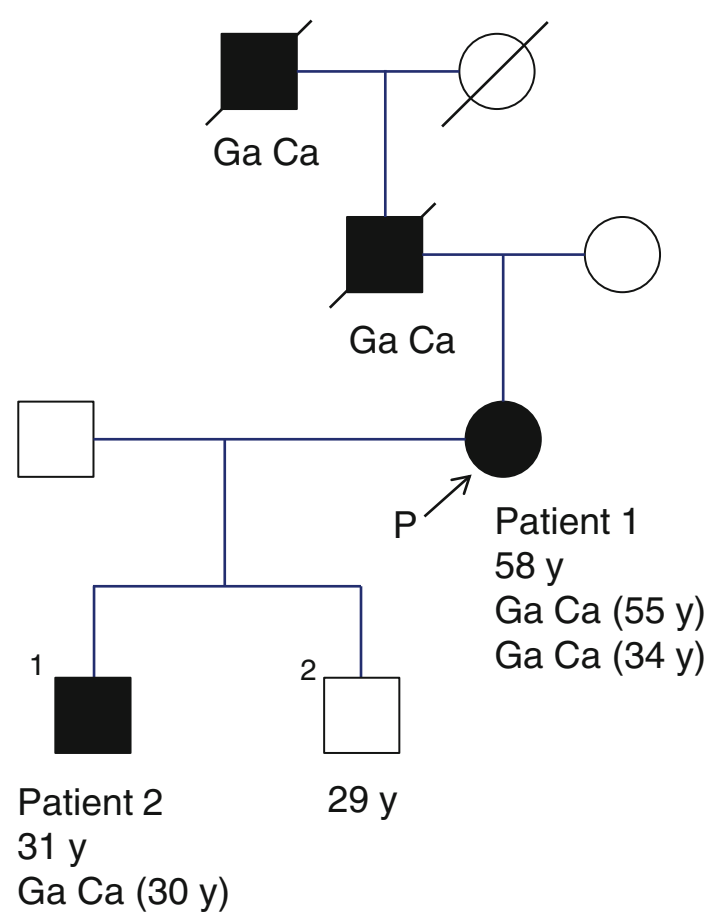

Fig. 1 Pedigree chart. The presence of the deletion mutation (Fig. 4) was confirmed in patients 1 and 2 in two generations
Immunohistochemistry was performed using anti-E-cadherin antibody (NCH-38; 1:100 dilution; DAKO, Glostrup, Denmark) as previously described [8]. The results showed loss of E-cadherin expression in SRCCs. (Fig. 2e). These histopathological findings were exactly identical to the findings in the HDGC cases reported by Guilford et al. [1]. No lymph node metastasis was observed.

Based on the pathological findings of the proband (patient 1), we suspected that she might have HDGC. Therefore, we performed surveillance endoscopy of two sons of the proband. Neither son had a history of malignancy. EGD for the 30-year-old elder son (Patient 2) detected three tiny pale areas at the body-antrum junction of the stomach (Fig. 3a, b). Apart from these tiny pale patches, no other endoscopic findings were suggestive of SRCC, but biopsy specimens obtained from each of the three pale areas revealed SRCC. Based on these findings, we clinically diagnosed HDGC in both patients, and total gastrectomy was performed on patient 2 . Histopathological assessment of the entire gastric mucosa revealed a total of 32 SRCCs (Fig. 3f) along with loss of E-cadherin expression in SRCCs (Fig. 3c, d, e). No apparent findings of recurrence or distant metastasis have been noted on the follow-up thus far. EGD in the younger son identified no significant endoscopic findings.

\section{The presence of a 275-kb deletion involving $\mathrm{CDH}$}

In order to perform genetic analysis, genomic DNA was extracted from peripheral leukocytes of patient 1, patient 2, and a healthy volunteer. Genomic DNA was also extracted from biopsy specimens obtained from the cancer site(s) in the stomachs of both patients. All 16 exons of $\mathrm{CDH} 1$ were sequenced by PCR-direct sequencing from both directions using previously reported primers [9]. Promoter methylation was analyzed by bisulfite modification and quantitative real-time methylation specific PCR (MSP) as previously described [10]. Primer sequences for the methylated DNA were $5^{\prime}$-TCG TTT TGG GGA GGG GTT C-3' (forward) and 5'-CAA ATA AAC CCC GAA AAC ACC G-3' (reverse), and those for the unmethylated DNA were 5'-GGA GGT ATT GTT TTT TGT ATT-3' (forward) and $5^{\prime}$-AAC AAA CCA TCA ACT CCA-3' (reverse). However, no $\mathrm{CDH} 1$ mutation or aberrant methylation was detected in peripheral lymphocytes or biopsy specimens of patient 1 .

Array-comparative genomic hybridization (CGH) analysis was performed according to the manufacturer's protocol using genomic DNA of peripheral leukocytes and human reference DNA (Caucasian, male \#5190-4370, female \#5190-4371, Agilent Technologies, Santa Clara, CA). DNA was digested with $A l u \mathrm{I}$ and $R s a \mathrm{I}$, labeled with $\mathrm{Cy} 5$ and $\mathrm{Cy} 3$, respectively, using a SureTag DNA 

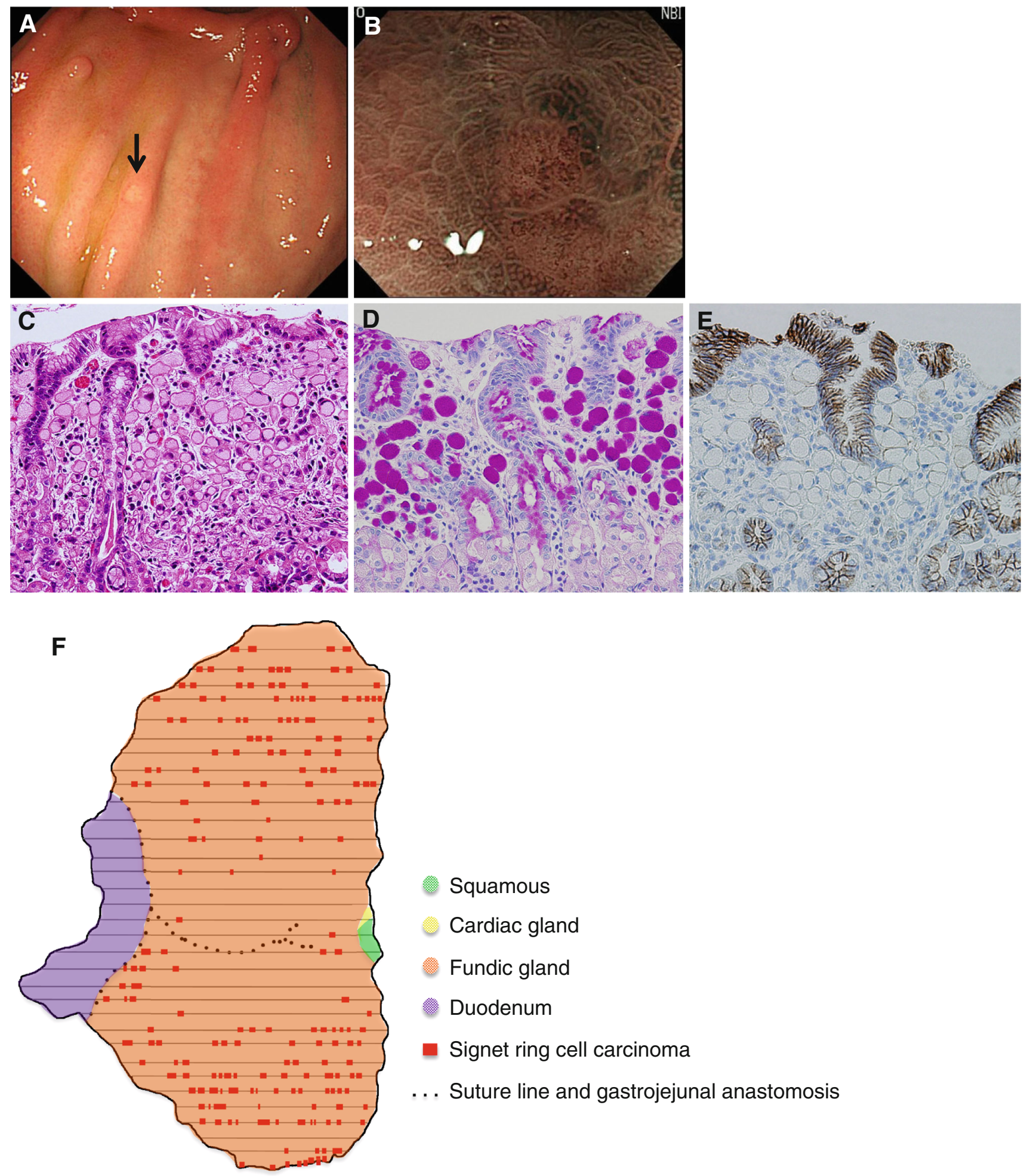

\section{Squamous}

Cardiac gland

Fundic gland

Duodenum

Signet ring cell carcinoma

... Suture line and gastrojejunal anastomosis

Fig. 2 Clinicopathological findings in patient 1. a Conventional endoscopy revealed pale lesions in the greater curvature of the remnant stomach (arrow). b Magnifying endoscopy with narrow-band imaging showed irregular microvessels. c Histology of the tumor showing SRCC proliferating in the upper layer of the fundic gland mucosa. d Periodic acid-Schiff (PAS) staining highlighted intracytoplasmic mucin in SRCC. e Immunohistochemistry for E-cadherin. Although normal gastric epithelial cells showed clear membrane staining, SRCCs showed loss of immunoreactivity. f Gastrectomy mapping study. Approximately 200 SRCC foci were observed in the resected specimen, predominantly near the greater curvature 

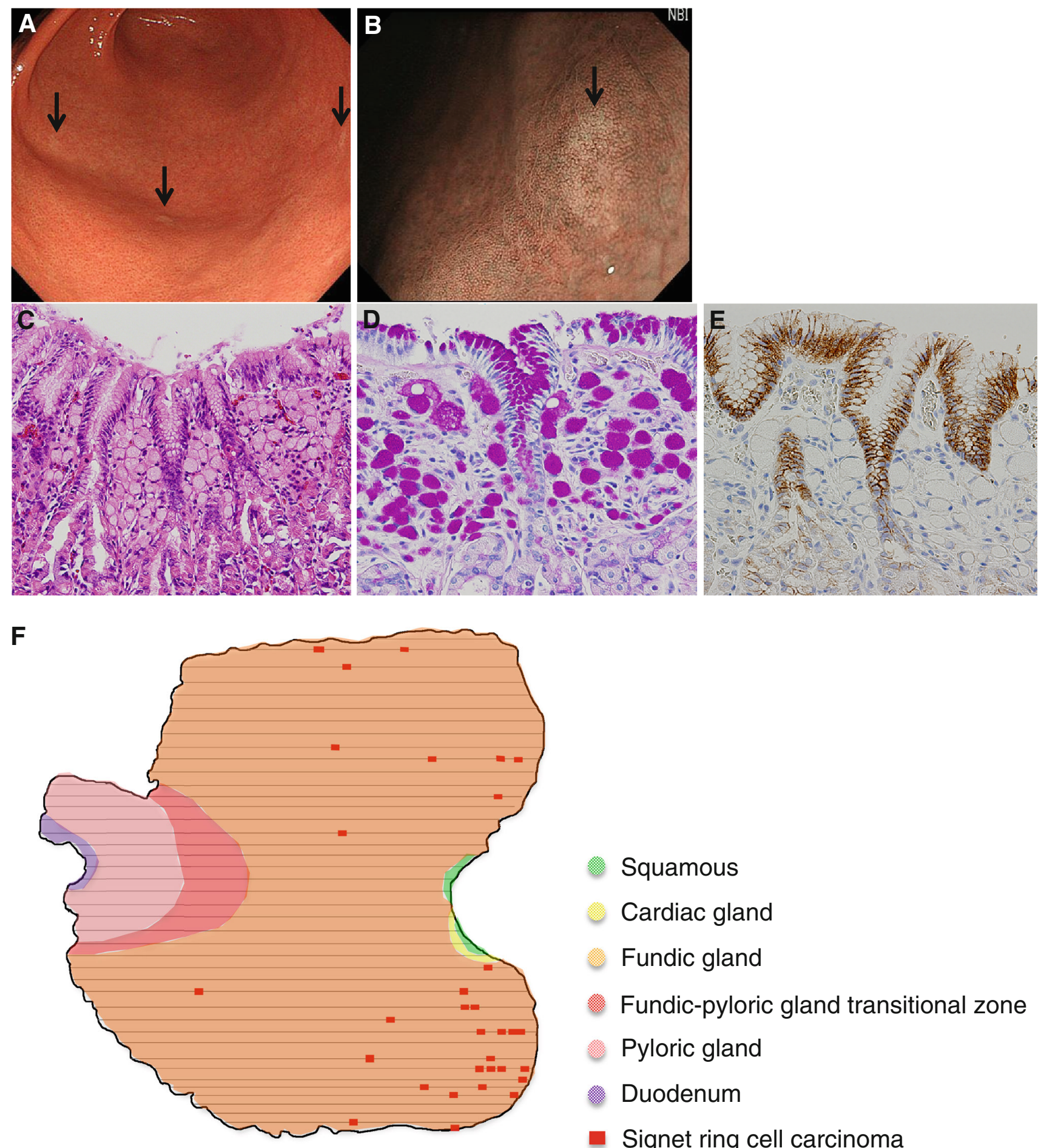

\section{Squamous}

\section{Cardiac gland}

\section{Fundic gland}

\section{Fundic-pyloric gland transitional zone}

\section{Pyloric gland}

\section{Duodenum}

\section{Signet ring cell carcinoma}

Fig. 3 Clinicopathological findings in patient 2. a Conventional endoscopy revealed pale lesions at the body-antrum junction of the stomach (arrows). b Magnifying endoscopy with narrow-band imaging. c Histological analysis showed SRCC in the fundic gland mucosa. d PAS staining highlighted intracytoplasmic mucin in SRCC.

Complete Labeling Kit (Agilent Technologies), and hybridized to a SurePrint G3 Cancer CGH + SNP $4 \times 180 \mathrm{~K}$ microarray (Agilent Technologies). The e Immunohistochemistry revealed lack of E-cadherin expression in SRCC. f Gastrectomy mapping study. A total of 32 signet ring cell carcinoma foci were detected exclusively in the fundic gland area. The finding was similar to that in patient 1

microarray was scanned with an Agilent G2565BA microarray scanner (Agilent Technologies), and the scanned data were processed using the Agilent CytoGenomics 
Fig. 4 A 275-kb deletion involving exons 7-16 of $\mathrm{CDH1}$. a Array CGH profiles obtained from peripheral leukocyte DNA of patient 1 , patient 2 , and a healthy control (33-year-old Japanese male without history of gastric cancer or family history of gastric cancer). The profiles of patients 1 and 2 showed a $275-\mathrm{kb}$ deletion. Deleted regions and the $C D H 1$ locus are shown by a green square and a red bar, respectively. A deletion of an exactly identical region was observed in both patients 1 and 2. b The location of the breakpoint within the $\mathrm{CDH} 1$ gene. The $5^{\prime}$-most probe of the deleted region (probe $y$, A_16_P03169109) was located on exon 7 , and the $3^{\prime}$-most probe of the intact region (probe x, A_16_P03169108) was located on exon 6. c Copy numbers of $C D H 1$ exons $4,6,7$, $8,10,13$, and 16 in patient 1 , Pptient 2, and a healthy control analyzed by TaqMan copy number assays. Deletion of CDH1 exons 7-16 was confirmed in patients 1 and 2 . The TaqMan probes for exons 4 , $6,7,8,10,13$, and 16 were Hs00296979_cn,

Hs01313772_cn,

Hs01453316_cn,

Hs00466712_cn,

Hs01438786_cn,

Hs01952025_cn, and

Hs02507752_cn, respectively.

For the seven exons above, copy numbers of patients 1 and 2 relative to a healthy control were calculated by $\Delta \Delta \mathrm{Ct}$ method

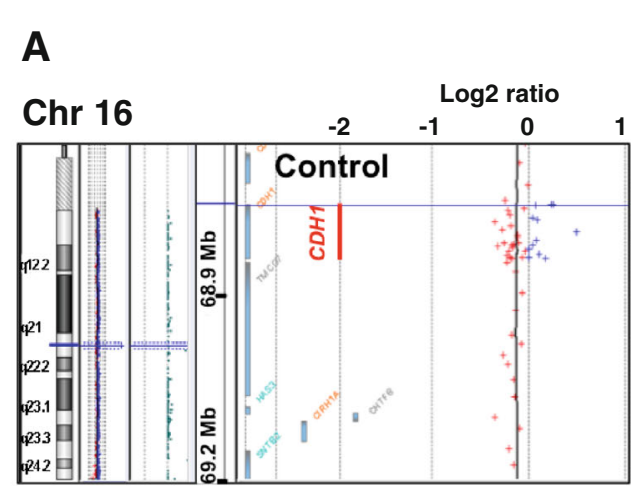

C

Exon

4

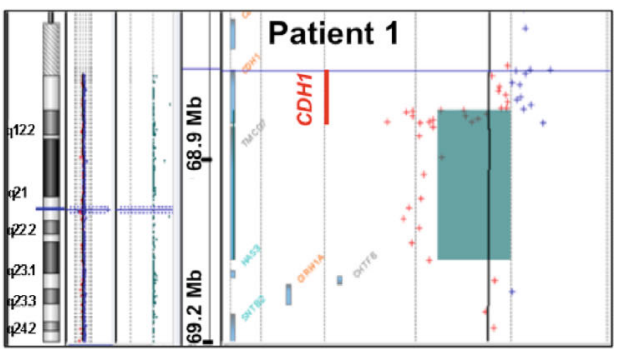

7

6

Control 1

Patient 2

Relative DNA copy number

0

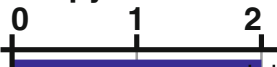

Patient 1

Patient

2
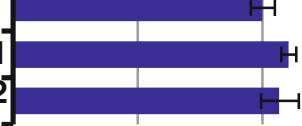

Control

7 Patient

Patient
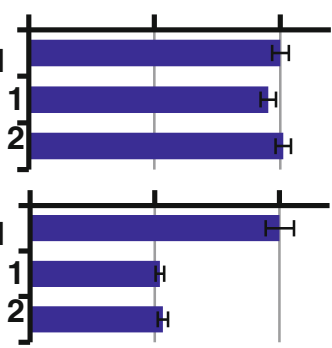

8

Control

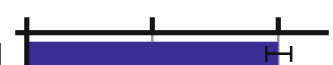

10

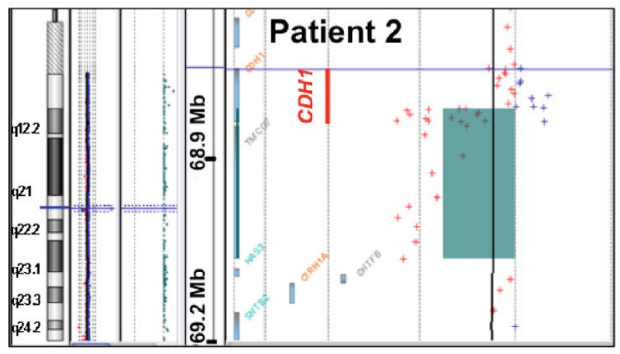

$13 \begin{aligned} & \text { Control } \\ & \text { Patient 1 } \\ & \text { Patient 2 }\end{aligned}$

B

Chromosome 16

$68,678,151$

$68,844,189 \quad 68,845,689$

$68,869,444$

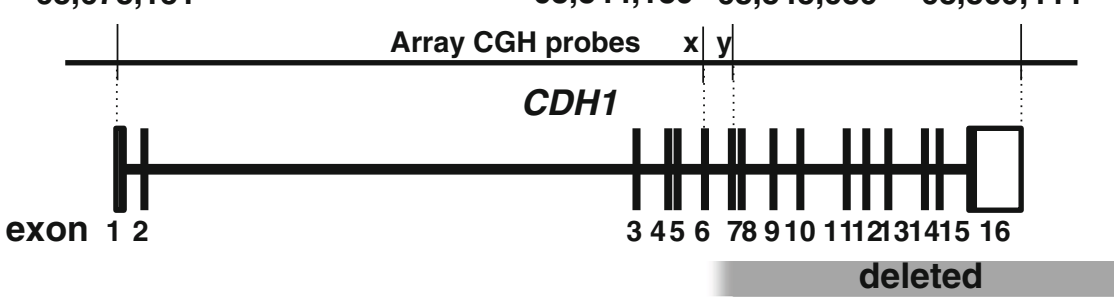

Software (Agilent Technologies). Regions that were covered by five probes or more and showed a mean $\log 2$ ratio $>0.5$ or $<-0.5$ were considered to have a gain or loss of the copy numbers, respectively. The CGH analysis revealed a 275-kb deletion in both patients 1 and 2 that involved exons 7-16 of $\mathrm{CDHl}$ (Fig. 4a, b). There were no other genomic regions that had altered copy numbers commonly in patients 1 and 2 but not in a Japanese healthy control (Supplementary Table 1). The deletion of exons 7-16 of $\mathrm{CDH} 1$ was further confirmed by copy number analysis of CDHI exon 4, 6, 7, 8, 10, 13, and 16 using TaqMan copy number assays (Life Technologies, Grand island, NY) (Fig. 4c).

\section{Discussion}

We presented a Japanese HDGC family with a $275-\mathrm{kb}$ deletion involving exons 7-16 of $\mathrm{CDH}$. From the initial EGD, we strongly suspected patient 1 to suffer from HDGC according to the clinical course and histopathological findings, which, although they did not fulfill the first 
diagnostic criteria for HDGC formulated by the International Gastric Cancer Linkage Consortium (IGCLC) in 1999 [11], fulfilled the criteria updated in 2009 [2], individuals with diffuse gastric cancer before the age of 40 with or without family history. We therefore recommended the proband's offspring to undergo EGD, and one son had three tiny pale areas at the body-antrum junction of the stomach that were confirmed to be SRCCs. Other than the tiny pale patches on endoscopy, there were no other endoscopic findings suggestive of SRCC. In the IGCLC guidelines, it is recommended that any endoscopically visible lesions should be targeted and that random sampling of six biopsies should additionally be taken for each of the following anatomical zones: antrum, transitional zone, body, fundus, and cardia. In total, a minimum of 30 biopsies is recommended. However, we were able to perform successful targeted biopsies of a minimal number of biopsy sites in patient $1(n=6)$ and $2(n=3)$ because good preparation was obtained as previously described [12]. Based on the presence of multiple SRCCs, patient 2 was also diagnosed with HDGC and was successfully treated with total gastrectomy.

The existence of numerous SRCCs strongly indicated that this family has HDGC. Although the expression of E-cadherin was absent or reduced in the tumor cells, we were unable to detect any small mutations in any exons of $\mathrm{CDH} 1$ or to detect aberrant methylation of $\mathrm{CDHl}$ in DNA collected from the peripheral leukocytes of the two patients. Generally, 25-30 \% of HDGC families are known to have germline mutations of the $\mathrm{CDHl}$ gene [2]; $77.9 \%$ of all reported $\mathrm{CDH} 1$ germline mutations are truncating mutations, and their locations span the whole length of the $\mathrm{CDHl}$ gene without any hot spots [13]. At the same time, $4 \%$ of reported $\mathrm{CDHl}$ germline mutations exhibit large deletions that are not detectable by conventional DNA sequencing [14]. Therefore, we continued to search for a large deletion of the $C D H 1$ gene in patients 1 and 2, and, by CGH analysis, we detected a pathogenic 275 -kb deletion in both patients 1 and 2. To our knowledge, this is the first case of HDGC harboring a large germline deletion in the range of hundreds of kilobases involving $\mathrm{CDH}$.

GCs have marked geographic variation in their incidence, with high incidences in East Asian countries and low incidences in Western countries. On the other hand, the majority of HDGC with $\mathrm{CDHI}$ mutations have been reported in the Western countries. To our knowledge, there have been only two Japanese HDCG families with germline $\mathrm{CDH} 1$ mutations reported so far [7]. However, it still remains unclear whether HDCG truly occurs less in Japan than in Western countries. The rarity of HDGC in Japan might be due to insufficient surveillance rather than reflecting the actual incidence. The high incidence of sporadic GCs is likely to increase "false-positive" familial
GCs and make it difficult to identify hereditary GCs. The IGCLC determined diagnostic criteria for HDGC, but these criteria are likely to be too broad to be used in areas with high GC incidence, including Japan [2, 11]. In addition, the prevalence of large deletions, as suggested by the study of Yamada et al. [7] and here, might have made detection of the $C D H 1$ mutation difficult.

Although HDGC has been regarded as rare in East Asia, these two recent reports show that there are HDGC families in East Asia and suggest the importance of the analysis of large deletions for cases with multiple SRCCs. Genetic counseling and testing may be advised for patients with multiple SRCCs and their family members even in East Asia.

\section{References}

1. Guilford P, Hopkins J, Harraway J, McLeod M, McLeod N, Harawira $\mathrm{P}$, et al. E-cadherin germline mutations in familial gastric cancer. Nature. 1998;392:402-5.

2. Fitzgerald RC, Hardwick R, Huntsman D, Carneiro F, Guilford P, Blair V, et al. Hereditary diffuse gastric cancer: updated consensus guidelines for clinical management and directions for future research. J Med Genet. 2010;47:436-44.

3. Park JG, Yang HK, Kim WH, Caldas C, Yokota J, Guilford PJ. Report on the first meeting of the International Collaborative Group on Hereditary Gastric Cancer. J Natl Cancer Inst. 2000;92: 1781-2.

4. Lynch HT, Grady W, Suriano G, Huntsman D. Gastric cancer: new genetic developments. J Surg Oncol 2005;90:114-33; discussion 33

5. Wang Y, Song JP, Ikeda M, Shinmura K, Yokota J, Sugimura H. Ile-Leu substitution (I415L) in germline E-cadherin gene (CDH1) in Japanese familial gastric cancer. Jpn J Clin Oncol. 2003;33: $17-20$.

6. Kim HC, Wheeler JM, Kim JC, Ilyas M, Beck NE, Kim BS, et al. The E-cadherin gene (CDH1) variants T340A and L599 V in gastric and colorectal cancer patients in Korea. Gut. 2000;47: $262-7$.

7. Yamada H, Shinmura K, Ito H, Kasami M, Sasaki N, Shima H, et al. Germline alterations in the $\mathrm{CDH} 1$ gene in familial gastric cancer in the Japanese population. Cancer Sci. 2011;102:1782-8.

8. Yamada M, Sekine S, Ogawa R, Taniguchi H, Kushima R, Tsuda $\mathrm{H}$, et al. Frequent activating GNAS mutations in villous adenoma of the colorectum. J Pathol. 2012;228:113-8.

9. Berx G, Cleton-Jansen AM, Nollet F, de Leeuw WJ, van de Vijver M, Cornelisse C, et al. E-cadherin is a tumour/invasion suppressor gene mutated in human lobular breast cancers. EMBO J. 1995;14:6107-15

10. Asada K, Ando T, Niwa T, Nanjo S, Watanabe N, Okochi-Takada $\mathrm{E}$, et al. FHL1 on chromosome $\mathrm{X}$ is a single-hit gastrointestinal tumor-suppressor gene and contributes to the formation of an epigenetic field defect. Oncogene. 2013;32:2140-9.

11. Caldas C, Carneiro F, Lynch HT, Yokota J, Wiesner GL, Powell SM, et al. Familial gastric cancer: overview and guidelines for management. J Med Genet. 1999;36:873-80.

12. Bhandari $P$, Green S, Hamanaka H, Nakajima T, Matsuda T, Saito Y, et al. Use of Gascon and Pronase either as a pre-endoscopic drink or as targeted endoscopic flushes to improve 
visibility during gastroscopy: a prospective, randomized, controlled, blinded trial. Scand J Gastroenterol. 2010;45:357-61.

13. Carneiro F, Oliveira C, Suriano G, Seruca R. Molecular pathology of familial gastric cancer, with an emphasis on hereditary diffuse gastric cancer. J Clin Pathol. 2008;61:25-30.
14. Oliveira C, Senz J, Kaurah P, Pinheiro H, Sanges R, Haegert A, et al. Germline CDH1 deletions in hereditary diffuse gastric cancer families. Hum Mol Genet. 2009;18:1545-55. 